\title{
Analysis of Economic Measurement Model Based on Approach of Social Computing
}

\author{
Peng Liang ${ }^{1 *}$, a Haochen Hua ${ }^{2, b}$ and Jian $\mathrm{Zhou}^{3}$ \\ ${ }^{1}$ College of Economics, Northwest Normal University, Lanzhou, China \\ ${ }^{2}$ Research Institute of Information Technology, Tsinghua University, Beijing, China \\ ${ }^{3}$ College of Electrical Engineering, Northwest Minzu University, Lanzhou, China \\ a765774952@qq.com, bhhchen@foxmail.com \\ *The corresponding author: Liang peng(765774952@qq.com)
}

Keywords: Economics measurement; Social computing; Distributed parallel

\begin{abstract}
The main purpose of this paper is to analyze the modern economic structure model based on approach of social computing and experimental modeling. A non-linear distributed parallel model is developed for the aim of further exploring the relation between the rate of economic growth and complex human behavior. Furthermore, one also finds a fact that the rate of economic growth is comprehensively affected by multi society elements. For instance, the greater the rigid demand of society, the stability of the parallel model would be. Overall, we hope our simulation results and analysis method could provide a new analytical pathway for guiding and enhancing the stability of economic growth and economic structure.
\end{abstract}

\section{Introduction}

The computational experiment is a step of calculating the simulation with the further development of computational techniques and analytical methods [1]. The study of the experimental computing has been attracted more and more relevant researchers, in particular in human behavior and economic modeling [2]. Of course, experimental computing does not require that the experimental phenomenon be the same as the actual occurrence. Furthermore, the artificial system approach does not preclude the approximation of objective systems, certain practical applications are still our future research goal $[3,4]$.

At present, the key idea of the artificial system modeling is the approach of agent-based [5,6], which can be provide a reference for comprehensively simulating actual society, such as, journal impact factor, migration of population, and investment analysis. Especially for certain complex systems, which involve the relation among society and human behavior. Artificial society-based artificial system approach is a meaningful attempt at present. In the distributed parallel model and system, the simulation results often have a strong subjectivity, uncontrollability and uncertainty. Thus, the final results have not universal $[7,8]$.

In this article, according to the approach of social computing and agent-based model $[9,10]$, a distributed parallel model is developed for analyzing the current economic setting and dynamical society elements $[11,12]$. In summary, we hope our analysis could provide a hints for further study, which combined the approach of social computing and economic modeling.

The rest of this paper is organized as follows: the distributed parallel model is elaborately described in Section 2. Section 3 endeavor to provide a satisfactory simulation result according to the actual data. Finally, this article is briefly concluded in Section 4.

\section{Economic Setting and Model Formulation}

When a new AS is being added into the economic network, it creates a certain number of edges connecting the existing economic structure. Namely, there might also appear newer 
interconnections with the already model nodes. Next we attempt to show how a distributed parallel model is used to structure a dynamical economic section. According to the sample data set, we hypothesize that the dynamical economic model could combined with the approach of social computing. Of course, it is worth noting that the simulation results are the part of future development, rather than a sample simulation experiment.

Define certain different geographic regions for the dynamic model. In each step, a new customer is added to the existing economic society along with nodes $m$ and $i$, the compute formulation as follows:

$$
\Pi\left(y_{i}\right)=\frac{y_{i}}{\sum_{j} y_{j}}
$$

Where $y_{i}$ is the open-degree (the number of open-pointing edges) of node $i$.

Start with first step of distributed parallel model, a new item for connection is not only a dynamic parallel system, but also a non-linear, the process of economic variation can be formulated as follows:

$$
\left\{\begin{array}{c}
\dot{x}_{1}(t)=a x_{1}(t)+f \sum_{1}^{N} \omega_{1 j}\left(x_{j}-x_{1}\right)+b v(t) \\
\dot{x}_{2}(t)=a x_{2}(t)+f \sum_{1}^{N} \omega_{2 j}\left(x_{j}-x_{2}\right)+b v(t) \\
\cdot \\
\cdot \\
\cdot \\
\dot{x}_{N}(t)=a x_{N}(t)+f \sum_{1}^{N} \omega_{N j}\left(x_{j}-x_{N}\right)+b v(t)
\end{array}\right.
$$

In this parallel system of equations, $x_{i}(t)$ is a structured virtual social community, $v(t)$ is the robust index of parallel system, and $\omega_{N j}$ is the degree of social purchasing powering. In addition, we note a fact that the node with the highest centroids are split, a customer at $i$ chooses $(i, j)$, which connects power $i$ and power $j$, as the next leg of travel according to the following preferential probability:

$$
\Pi=\frac{\omega_{i j}}{\sum_{j \in \Gamma_{i}} \omega_{i j}}
$$

Calculating the first and the second moments of $f_{i}$ yields

$$
\left\langle f_{i}\right\rangle=\sum_{n=0}^{\infty} n P_{i}(n)=\frac{s_{i}}{\sum_{i=1}^{N} R M}
$$

This calculation indicates that the number of customer and the degree of purchasing could be divided into 4 steps. Now, suppose $P_{i}(n)>0$, namely, a series of rigid index, which are the stability and dynamic of society, vitality of market and so on. Surely, we should aware a fact that the multi elements could be consisted in certain dubious factors. Therefore, the main aim of distributed parallel model is to provide an analytical method, rather than reproduce the actual situation of society. The simulation results are illustrated in Tab. 1: 
Table 1 Relation between certain elements and category of model

\begin{tabular}{cccc}
\hline No. & Time (months) & Parallel Degree & Dynamical Model \\
\hline 1 & 5 & 1.0012 & Distributed Parallel \\
2 & 10 & 1.1300 & Distributed Parallel \\
3 & 15 & -1.1125 & Agent-Based \\
4 & 20 & 1.2362 & Distributed Parallel \\
5 & 25 & -1.1050 & Agent-Based \\
6 & 30 & 1.1255 & Distributed Parallel \\
\hline
\end{tabular}

\section{Simulation and Analysis}

Here based on above formulation of distributed parallel model, we simulate the relation between time (months) and economic growth rate, see Fig. 1.

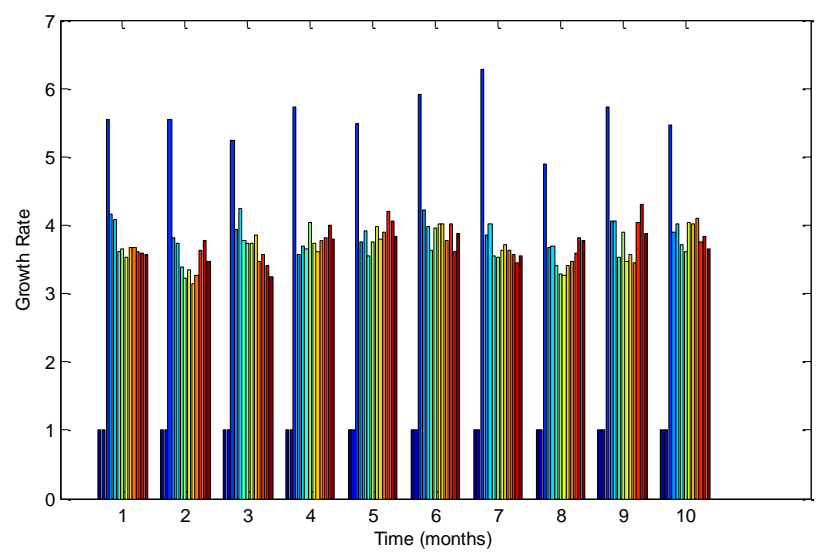

Figure 1. Relation between time and economic growth rate

Suppose that the life time is linear and increasing, the slope of growth rate $(\alpha)$ is always increasing. In order to achieve this objective, we randomly select 300 human society communities for the purpose of analyzing different actual situation. In fact, the initial aim of this section is to numerically and qualitatively verify former dynamical parallel model. Subsequently, we would show how the degree of economic structure is influenced by parameter values, or in other words, testing the reliability of parameter settings, the specific steps are as follows:

Step1: Based on dynamic parallel system of urban and rural labor transfer model.

Step2: Gives a method based on social computing parallel system cluster synchronization conditions.

Step3: Put forward a kind of distributed clustering algorithm and model.

The simulation result, which is relation between time (year) and impulse degree (\%) as intuitively shown in Fig. 2. It should be explained that the Gaussian noise of the parallel model has already been filtered, in particular when the time reaches to year 2010.

In fact, current research on social computing issues, the focus of the study is mainly consisted in the assumption and rationality of the proposed model. Further speaking, the analysis and evaluation of computing experimental and social computing has increasingly step into existent modeling. 


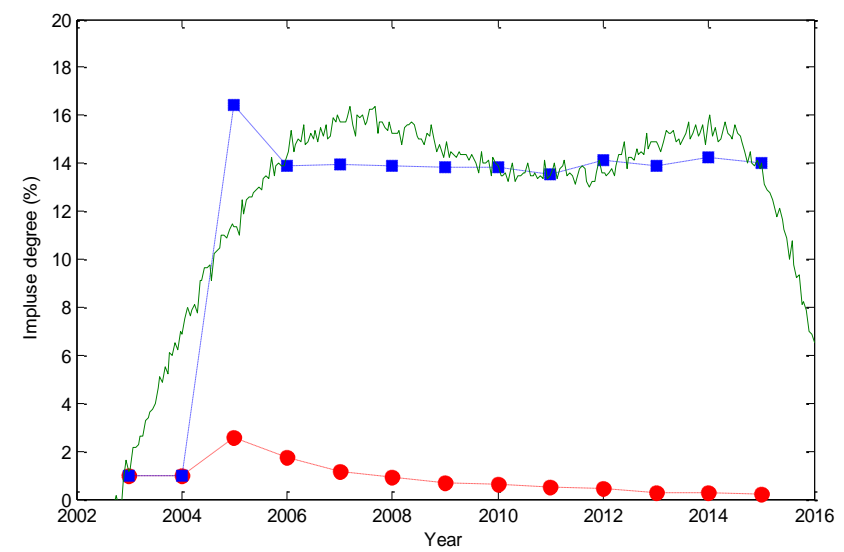

Figure 2. Relation between time (year) and impulse degree (\%)

From the above curve, it is easily to find that the open-degree and rigid demand of society might jointly affected by the impulse-degree. Alternatively, our model could also show how the impulse degree to partly stimulate the stability of the virtual society community.

\section{Conclusions and Discussion}

The aim is to reveal the underlying mechanism of human opinion formation, evolution and convergence, hoping to gain a better understanding of human social behaviors particularly their opinion dynamics. The stability of economic parallel model is partly influenced by multi society elements and human behaviors. For instance, the purchasing powering of customer and the rigid demand of different communities all have a comprehensive impulse for current economic structure, which can be viewed a complex artificial system, or in other words, we should conduct present research direction to further explore the relation between the complex human behaviors and economic society setting.

\section{References}

[1] F. Y. Wang: IEEE Intelli. Syst. Vol. 26 (2011) No.1, p.2-4.

[2] N. Cai, J. W. Cao, H. Y. Ma and C. X. Wang: Arab J Sci Eng, Vol. 39 (2014) No.3, pp. 2427-2434.

[3] N. Cai, M. He, Q. Wu and M. J. Khan: J. Syst. Sci. Complexity, DOI: 10.1007/s11424-017-6273-7.

[4] Y. Q. Guan, Z. J. Ji, L. Zhang and L. Wang: Int. J. Robust Nonlin. Control, DOI: 10.1002/rnc.3798.

[5] Z. J. Ji, H. Lin and H. S. Yu: IEEE Trans. Automat. Control, Vol. 60 (2015) No.3, pp.781-786.

[6] F. Y. Wang: IEEE Intelli. Syst., Vol. 22 (2007) No.5, p.65-67.

[7] X. Huang, J. Gao, S. V. Buldyrev, S. Havlin and H. E. Stanley: Physical Review E, Vol. 83 (2011) No.6, 065101.

[8] N. Cai, C. Diao and M. J. Khan: Complexity, 4978613, 2017.

[9] J. X. Xi, M. He, H. Liu and J. F. Zheng: J Franklin Inst, Vol. 353 (2016) No.16, pp.4074-4090.

[10] R. Carvalho, L. Buzna, F. Bono, E. Guti'errez, W. Just and D. Arrowsmith: Physical Review E, Vol. 80 (2009) No.2, 016106.

[11] N. Cai, M. J. Khan: Int. J. Control Automat. Syst., Vol. 15 (2017) No.1, pp.169-177.

[12]Z. J. Ji and H. S. Yu: IEEE Trans. Cybernet., DOI: 10.1109/TCYB.2016.2549034. 